\title{
MEMS-based inertial navigation based on a magnetic field map
}

\author{
Manon Kok, Niklas Wahlström, Thomas Schön and Fredrik Gustafsson
}

\section{Linköping University Post Print}

\section{Tweet}

N.B.: When citing this work, cite the original article.

(C) 2013 IEEE. Personal use of this material is permitted. Permission from IEEE must be obtained for all other users, including reprinting/ republishing this material for advertising or promotional purposes, creating new collective works for resale or redistribution to servers or lists, or reuse of any copyrighted components of this work in other works.

Original Publication:

Manon Kok, Niklas Wahlström, Thomas Schön and Fredrik Gustafsson, MEMS-based inertial navigation based on a magnetic field map, 2013, Proceedings of the 38th International Conference on Acoustics, Speech, and Signal Processing (ICASSP), 6466-6470.

http://dx.doi.org/10.1109/ICASSP.2013.6638911

Postprint available at: Linköping University Electronic Press http://urn.kb.se/resolve?urn=urn:nbn:se:liu:diva-102632 


\title{
MEMS-BASED INERTIAL NAVIGATION BASED ON A MAGNETIC FIELD MAP
}

\author{
Manon Kok, Niklas Wahlström, Thomas B. Schön, Fredrik Gustafsson \\ Division of Automatic Control, Linköping University, Linköping, Sweden \\ \{manko, nikwa, schon, fredrik\}@isy.liu.se
}

\begin{abstract}
This paper presents an approach for $6 \mathrm{D}$ pose estimation where MEMS inertial measurements are complemented with magnetometer measurements assuming that a model (map) of the magnetic field is known. The resulting estimation problem is solved using a RaoBlackwellized particle filter. In our experimental study the magnetic field is generated by a magnetic coil giving rise to a magnetic field that we can model using analytical expressions. The experimental results show that accurate position estimates can be obtained in the vicinity of the coil, where the magnetic field is strong.
\end{abstract}

Index Terms - Magnetic field, inertial navigation, state estimation, Rao-Blackwellized particle filter, magnetometer.

\section{INTRODUCTION}

With the reducing cost of accelerometers and gyroscopes (inertial sensors) and magnetometers, these sensor are becoming increasingly available in day-to-day life. It is for instance common that these sensors are present in modern smartphones. Positioning based on inertial sensors alone suffers greatly from drift and does not give reliable estimates for any but the highest quality sensors. Because of this, sensors such as GPS and ultra-wideband are often used as an aiding source [1]. While GPS solutions only work for outdoor applications, indoor solutions are often highly dependent on additional infrastructure.

Magnetometers are a reliable source of information due to their high sampling rates and reliable sensor readings. They measure the superposition of the local earth magnetic field and the magnetic field induced by magnetic structures in the vicinity. Magnetometers are widely used as a source of heading information, relying on the assumption that no magnetic disturbances are present. Especially in indoor applications this assumption is often violated due to the presence of steel in the construction of buildings and objects like radiators, tables and chairs.

This paper presents a method to obtain accurate position and orientation estimates based on inertial and magnetometer data assuming a map of the magnetic field is known. This enables positioning with widely available sensors, without requirements on additional infrastructure.

In recent years, the idea of using the presence of magnetic disturbances as a source of position information has started appearing in the literature. Most interest is from the robot localization perspective where odometry information is available $[2,3,4,5]$. Generally, in these applications localization is only considered in $2 \mathrm{D}$, and the sensor is assumed to be rotating around only one axis. To the best of the authors' knowledge, little work has been done on combining inertial and magnetometer measurements, for example [6, 7]. This is a more challenging problem compared to using odometry information, since low grade inertial measurement units (IMUs) generally have poor dead-reckoning performance. The approach presented in [6]

This work is supported by MC Impulse, a European Commission, FP7 research project and CADICS, a Linneaus Center funded by the Swedish Research Council (VR).

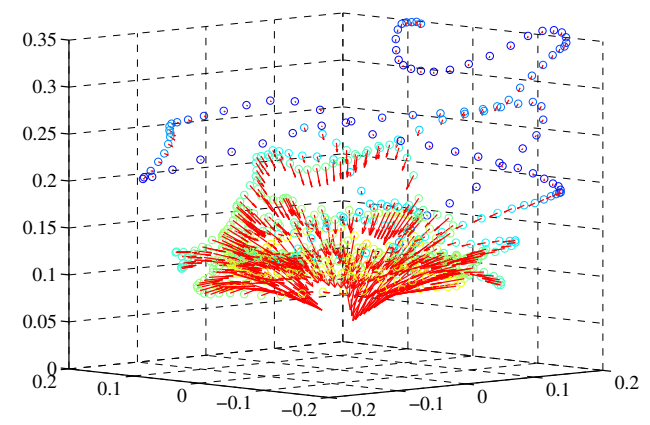

Fig. 1. Magnetometer measurements represented in the earth coordinate frame. The measurements have been preprocessed by subtracting the earth magnetic field. The magnitude is indicated by the colors and the direction by the arrows.

is not based on magnetic field maps, but uses knowledge about the physical properties of the magnetic field and its gradient to aid localization using an extended Kalman filter approach. Other approaches focus on using sensors in smartphones for localization $[8,9,10]$ and consider magnetometer data only or very limited information from the inertial sensors. The direction of the magnetic field can, however, only be derived from the magnetic field measurements when the sensor orientation is known. Not estimating the full orientation therefore poses constraints on the allowed sensor rotations. In our approach no constraints on the sensor rotations are required since the full $6 \mathrm{D}$ pose is estimated.

To isolate the problem of localization inside a known magnetic field map from the problem of obtaining the map, this work assumes that the magnetic field map is known and is generated by a magnetic coil. The reason for using a magnetic coil is that it is one of the few cases for which the magnetic field can be computed analytically. In other words, we have a perfect model describing the magnetic field produced by the magnetic coil. The magnetic field measurements can be described as a nonlinear function of the sensor position in this map and its orientation with respect to the map.

\section{MODELS}

Before introducing the dynamic and measurement equations, the relevant coordinate frames and the state vector will be introduced. All measurements are assumed to be obtained in the body coordinate frame denoted by $b$, which is the coordinate frame of the measurement unit with the origin in the center of the accelerometer triad. The position is tracked in the earth coordinate frame denoted by $e$, which is fixed in the world. The magnetic field map is represented in the map coordinate frame denoted by $m$ whose orientation is assumed to be aligned with that of the coil. The origin of the earth coordinate frame $e$ is assumed to coincide with that of the map coordinate frame and with the center of the magnetic coil. 
The relevant state vector consists of the sensor's position $p^{\mathrm{e}}$ and velocity $v^{\mathrm{e}}$, its orientation with respect to the earth frame expressed as a unit quaternion $q^{\mathrm{eb}}=\left(\begin{array}{llll}q_{0} & q_{1} & q_{2} & q_{3}\end{array}\right)^{\top}$ and the gyroscope bias $b_{\omega}^{\mathrm{b}}$. In our model we have used the inertial measurements as inputs to the dynamic equations in order to not increase the state dimension. For reasons that will become clear after the model has been provided, we split the state vector into two parts

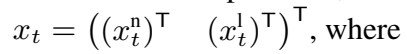

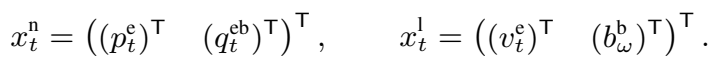

\subsection{Dynamical model}

The dynamical equations can be derived by using the inertial measurements as inputs. A commonly used, slowly time-varying random walk model is used for the gyroscope bias [1]. This leads to the following state update equations for the linear and nonlinear states $[1,11]$

$$
\begin{aligned}
& x_{t+1}^{\mathrm{n}}=\underbrace{\left(\begin{array}{cc}
\mathcal{I}_{3} & 0 \\
0 & \mathcal{I}_{4}
\end{array}\right)}_{A^{\mathrm{nn}}} x_{t}^{\mathrm{n}}+\underbrace{\left(\begin{array}{cc}
T \mathcal{I}_{3} & 0 \\
0 & -\frac{T}{2} \widetilde{S}\left(q_{t}^{\mathrm{eb}}\right)
\end{array}\right)}_{A_{t}^{\mathrm{nl}}\left(x_{t}^{\mathrm{n}}\right)} x_{t}^{1}+ \\
& \underbrace{\left(\begin{array}{ccc}
\frac{T^{2}}{2} \mathcal{R}\left(q_{t}^{\mathrm{eb}}\right) & \frac{T^{2}}{2} \mathcal{I}_{3} & 0 \\
0 & 0 & \frac{T}{2} \widetilde{S}\left(q_{t}^{\mathrm{eb}}\right)
\end{array}\right)}_{B_{t}^{\mathrm{n}}\left(x_{t}^{\mathrm{n}}\right)} u_{t}+ \\
& \underbrace{\left(\begin{array}{cc}
\frac{T^{2}}{2} \mathcal{R}\left(q_{t}^{\mathrm{eb}}\right) & 0 \\
0 & \frac{T}{2} \widetilde{S}\left(q_{t}^{\mathrm{eb}}\right)
\end{array}\right)}_{G_{t}^{\mathrm{n}}\left(x_{t}^{\mathrm{n}}\right)} \underbrace{\left(\begin{array}{c}
w_{a, t}^{\mathrm{b}} \\
w_{\omega, t}^{\mathrm{b}}
\end{array}\right)}_{w_{t}^{\mathrm{n}}} \\
& x_{t+1}^{1}=\underbrace{\left(\begin{array}{cc}
\mathcal{I}_{3} & 0 \\
0 & \mathcal{I}_{3}
\end{array}\right)}_{A^{\mathrm{Il}}} x_{t}^{1}+\underbrace{\left(\begin{array}{ccc}
T \mathcal{R}\left(q_{t}^{\mathrm{eb}}\right) & T \mathcal{I}_{3} & 0 \\
0 & 0 & 0
\end{array}\right)}_{B_{t}^{\mathrm{l}}\left(x_{t}^{\mathrm{n}}\right)} u_{t}+ \\
& \underbrace{\left(\begin{array}{cc}
T \mathcal{R}\left(q_{t}^{\mathrm{eb}}\right) & 0 \\
0 & \mathcal{I}_{3}
\end{array}\right)}_{G_{t}^{\mathrm{l}}\left(x_{t}^{\mathrm{n}}\right)} \underbrace{\left(\begin{array}{c}
w_{a, t}^{\mathrm{b}} \\
w_{\mathrm{b}_{\omega}, t}^{\mathrm{b}}
\end{array}\right)}_{w_{t}^{1}} .
\end{aligned}
$$

Here, $\mathcal{I}_{k}$ denotes the identity matrix of size $k \times k, \mathcal{R}\left(q_{t}^{\mathrm{eb}}\right) \in S O(3)$ is the rotation matrix obtained from the unit quaternion $q_{t}^{\mathrm{eb}}$ and ${ }^{1}$

$$
\widetilde{S}\left(q_{t}^{\mathrm{eb}}\right)=\left(\begin{array}{ccc}
-q_{1} & -q_{2} & -q_{3} \\
q_{0} & -q_{3} & q_{2} \\
q_{3} & q_{0} & -q_{1} \\
-q_{2} & q_{1} & q_{0}
\end{array}\right)
$$

The input vector $u_{t}$ is given by

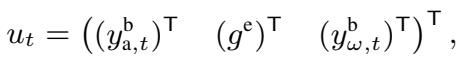

where $g^{\mathrm{e}}$ denotes the gravity vector and the accelerometer and the gyroscope measurements are denoted by $y_{\mathrm{a}}^{\mathrm{b}}$ and $y_{\omega}^{\mathrm{b}}$, respectively. The latter are modeled as

$$
\begin{aligned}
y_{\mathrm{a}, t}^{\mathrm{b}} & =R_{t}^{\mathrm{be}}\left(a_{t}^{\mathrm{e}}-g^{\mathrm{e}}\right)+w_{\mathrm{a}, t}^{\mathrm{b}}, \\
y_{\omega, t}^{\mathrm{b}} & =\omega_{t}^{\mathrm{b}}+b_{\omega}^{\mathrm{b}}+w_{\omega, t}^{\mathrm{b}},
\end{aligned}
$$

\footnotetext{
${ }^{1}$ Note that the propagation of the quaternion state in this way is an approximation, valid only for high sampling rates. The algorithm does not prevent use of the exact update equation and the approximation is only used to reduce computational complexity.
}

based on the fact that the accelerometer measures both the gravity vector and the body's free acceleration. The noise is modeled as

$$
\begin{aligned}
w_{\mathrm{a}}^{\mathrm{b}} & \sim \mathcal{N}\left(0, Q_{\mathrm{a}}\right), & Q_{\mathrm{a}} & =\sigma_{\mathrm{a}}^{2} \mathcal{I}_{3}, \\
w_{\omega}^{\mathrm{b}} & \sim \mathcal{N}\left(0, Q_{\omega}\right), & Q_{\omega} & =\sigma_{\omega}^{2} \mathcal{I}_{3}, \\
w_{\mathrm{b}_{\omega}}^{\mathrm{b}} & \sim \mathcal{N}\left(0, Q_{\mathrm{b}_{\omega}}\right), & Q_{\mathrm{b}_{\omega}} & =\sigma_{\mathrm{b}_{\omega}}^{2} \mathcal{I}_{3} .
\end{aligned}
$$

The state noise is assumed to be distributed according to

$$
\begin{aligned}
& w_{t}=\left(\begin{array}{c}
w_{t}^{\mathrm{n}} \\
w_{t}^{\mathrm{l}}
\end{array}\right) \sim \mathcal{N}(0, Q), \\
& Q=\left(\begin{array}{cc}
Q^{\mathrm{nn}} & Q^{\mathrm{nl}} \\
\left(Q^{\mathrm{nl}}\right)^{\top} & Q^{\mathrm{ll}}
\end{array}\right)=\left(\begin{array}{cc:cc}
Q_{\mathrm{a}} & 0 & Q_{\mathrm{a}} & 0 \\
0 & Q_{\omega} & 0 & 0 \\
\hdashline Q_{\mathrm{a}}^{\top} & 0 & Q_{\mathrm{a}} & 0 \\
0 & 0 & 0 & Q_{\mathrm{b}_{\omega}}
\end{array}\right) .
\end{aligned}
$$

Note that the linear and nonlinear state noise is highly correlated since the accelerometer noise acts on both the position and velocity states. This needs to be taken into account in the implementation.

\subsection{Magnetometer measurement model}

The magnetometer measurements are modeled as

$$
y_{\mathrm{m}, t}^{\mathrm{b}}=h\left(x_{t}^{\mathrm{n}}\right)+e_{\mathrm{m}, t}^{\mathrm{b}},
$$

where $e_{\mathrm{m}, t}^{\mathrm{b}} \sim \mathcal{N}(0, R)$ and $h\left(x_{t}^{\mathrm{n}}\right)$ is a function of the position $p_{t}^{\mathrm{e}}$ and orientation $q_{t}^{\mathrm{eb}}$ states. In practice this will be a superposition of the local earth magnetic field and all magnetic disturbances present.

As discussed in the introduction, to isolate the problem of positioning inside a map from the problem of making the map, we chose an experimental setup where the magnetic field is generated by a magnetic coil. In this case a magnetic field map is analytically known assuming the coil's position and orientation are known. The function $h\left(x_{t}^{\mathrm{n}}\right)$ is given by

$$
h\left(x_{t}^{\mathrm{n}}\right)=\mathcal{R}\left(q^{\mathrm{be}}\right) R^{\mathrm{em}} B\left(R^{\mathrm{me}} p_{t}^{\mathrm{e}}\right) .
$$

The function $B\left(R^{\mathrm{me}} p_{t}^{\mathrm{e}}\right)$ gives the magnetic field in the map coordinate frame at a position $p^{\mathrm{m}}$. The expression for the magnetic field from the coil is given by [12]

$$
\begin{aligned}
B\left(p^{\mathrm{m}}\right) & =\frac{\mu_{0} N_{w} I}{2 \pi \sqrt{\left(\sqrt{p_{x}^{2}+p_{y}^{2}}+a\right)^{2}+p_{z}^{2}}} \cdot \\
& \left(\begin{array}{c}
\frac{p_{x} p_{z}}{p_{x}^{2}+p_{y}^{2}}\left[-K(k)+\frac{a^{2}+p_{x}^{2}+p_{y}^{2}+p_{z}^{2}}{\left(\sqrt{p_{x}^{2}+p_{y}^{2}}-a\right)^{2}+p_{z}^{2}} E(k)\right] \\
\frac{p_{y} p_{z}}{p_{x}^{2}+p_{y}^{2}}\left[-K(k)+\frac{a^{2}+p_{x}^{2}+p_{y}^{2}+p_{z}^{2}}{\left(\sqrt{p_{x}^{2}+p_{y}^{2}}-a\right)^{2}+p_{z}^{2}} E(k)\right] \\
{\left[K(k)+\frac{a^{2}-p_{x}^{2}-p_{y}^{2}-p_{z}^{2}}{\left(\sqrt{p_{x}^{2}+p_{y}^{2}}-a\right)^{2}+p_{z}^{2}} E(k)\right]}
\end{array}\right)
\end{aligned}
$$

where $p^{\mathrm{m}}=\left(\begin{array}{lll}p_{x} & p_{y} & p_{z}\end{array}\right), \mu_{0}$ is the magnetic permeability in vacuum, $a$ is the coil radius, $N_{w}$ is the number of windings, $I$ is the current through the coil and $E(k)$ and $K(k)$ are given by the following elliptic integrals

$$
\begin{aligned}
& E(k)=\int_{0}^{\pi / 2} \sqrt{1-k^{2} \sin ^{2} \theta} d \theta, \\
& K(k)=\int_{0}^{\pi / 2} \frac{1}{\sqrt{1-k^{2} \sin ^{2} \theta}} d \theta,
\end{aligned}
$$


where

$$
k=\sqrt{\frac{4 a \sqrt{p_{x}^{2}+p_{y}^{2}}}{\left(\sqrt{p_{x}^{2}+p_{y}^{2}}+a\right)^{2}+p_{z}^{2}}} .
$$

These equations implicitly assume that the origin of the earth coordinate frame coincides with that of the map coordinate frame. Note that our measurement model assumes that no background field is present.

\subsection{Some additional words about the magnetic field model}

The magnetic field of a coil is generally described as a function of the perpendicular distance $p_{z}$ towards the coil and the radial distance $r=\sqrt{p_{x}^{2}+p_{y}^{2}}$ towards the center of the coil $[12,13]$. However, in tracking we are interested in absolute position rather than just the distance to a source. Parametrizing the magnetic field in terms of a position $p_{x}, p_{y}, p_{z}$ introduces unobservability. Assuming the coil is placed horizontally, this results in two horizontal circles, one above and one below the coil, where the horizontal position is coupled to the heading as an unobservable manifold. We assume that the sensor can only be positioned above the coil and therefore have an entire circle of solutions at each time step. Note that in the more general case where multiple magnetic sources are present and possibly rotated with respect to each other, the unobservable manifold will be differently shaped or in some cases non-existent. To make our dynamic model applicable to any magnetic field map, we have not adapted the parametrization of our state vector to this specific structure.

\section{COMPUTING THE ESTIMATE}

As can be seen from the dynamical and measurement model presented in Section 2, the state dynamics is assumed to be linear while the measurement model is a nonlinear function of the sensor's position and orientation. A nonlinear filtering technique is therefore needed to compute a state estimate. A linear substructure can, however, be recognized, which can be exploited using a RaoBlackwellized particle filter (RBPF) in which the state is split into a state $x^{1}$ that enters linearly in both the dynamic and measurement model and a state $x^{\mathrm{n}}$ that enters non-linearly, where $x^{1}$ and $x^{\mathrm{n}}$ are defined by (1). An RBPF solves the nonlinear filtering problem by using a Kalman filter (KF) for the linear states and a particle filter (PF) for the nonlinear states.

The RBPF in this paper has been derived from [11] and [14] and is summarized in Algorithm 1. It applies the model structure (2), (8), the noise assumptions (6) and their correlations given in (7). In (13), $\bar{x}_{t}^{i}$ and $\bar{P}_{t}^{i}$ are computed, which are a stacked version of the nonlinear and linear states and covariances. Based on these, the nonlinear and linear time update are given by (14), (15) respectively. Note that in (15) the pseudo-inverse, denoted by $\dagger$, of $\bar{P}_{t}^{\mathrm{nn}, i}$ needs to be taken because this matrix is rank deficient due to the presence of quaternion states.

Since the measurement model (9) only depends on the nonlinear states, measurement information about the linear states is in our problem only available through the nonlinear states. Algorithm 1 does therefore not contain an explicit KF measurement update. However, measurement information implicitly present in the nonlinear states is taken into account in the linear states in (15).

\subsection{RBPF-MAP}

To compare particle filter estimates to reference data, a point estimate needs to be computed at each time step. The most commonly used approach for this is to take the conditional mean estimate. Due to the unobservability in our model (see Section 2.3), however, all particles on a horizontal circle are equally likely, which can lead to an uninformative point estimate in center of the circle.
Algorithm 1 Rao-Blackwellized particle filter

1. Initialization: For $i=1, \ldots, N$ generate $x_{0}^{\mathrm{n}, i} \sim p_{x_{0}^{\mathrm{n}}}$, set $\left\{x_{0}^{1, i}, P_{0}^{i}\right\}=\left\{x_{0}^{1}, P_{0}\right\}, \gamma_{-1}^{i}=\frac{1}{N}$, and set $t=0$.

2. Measurement update: For $i=1, \ldots, N$ evaluate the particle importance weights $\gamma_{t}^{i}=\frac{1}{c_{t}} \gamma_{t-1}^{i} p\left(y_{t} \mid x_{0: t}^{\mathrm{n}, i}, y_{0: t-1}\right)$ based on (8) where $c_{t}=\sum_{i=1}^{N} \gamma_{t-1}^{i} p\left(y_{t} \mid x_{0: t}^{\mathrm{n}, i}, y_{0: t-1}\right)$.

3. If $t>0$, compute the estimate $\widehat{x}_{t}$ based on (17).

4. Resampling: If $\widehat{N}_{\text {eff }}=\frac{1}{\sum_{i=1}^{N}\left(\gamma_{t}^{i}\right)^{2}}<\frac{2}{3} N$, resample $N$ particles with replacement from the set $\left\{x_{t}^{\mathrm{n}, i}, x_{t}^{1, i}\right\}_{i=1}^{N}$ where the probability to take sample $i$ is $\gamma_{t}^{i}$, and reset the weights to $\gamma_{t}^{i}=\frac{1}{N}$.

5. Time update: Determine the Gaussian mixture

$$
\begin{aligned}
& \bar{x}_{t+1}^{i}=A_{t}^{i} x_{t}^{i}+B_{t}^{i} u_{t}, \\
& \bar{P}_{t+1}^{i}=A_{t}^{1, i} P_{t}^{i}\left(A_{t}^{1, i}\right)^{\top}+G_{t}^{i} Q\left(G_{t}^{i}\right)^{\top},
\end{aligned}
$$

where

$$
\begin{aligned}
& \bar{x}_{t}^{i}=\left(\begin{array}{c}
\bar{x}_{t}^{\mathrm{n}, i} \\
\bar{x}_{t}^{1, i}
\end{array}\right), \\
& \bar{P}_{t}^{i}=\left(\begin{array}{cc}
\bar{P}_{t}^{\mathrm{nn}, i} & \bar{P}_{t}^{\mathrm{nl}, i} \\
\left(\bar{P}_{t}^{\mathrm{nl}, i}\right)^{\top} & \bar{P}_{t}^{\mathrm{ll}, i}
\end{array}\right), \\
& A_{t}^{\mathrm{l}, i}=\left(\begin{array}{c}
A_{t}^{\mathrm{nl}, i}\left(x_{t}^{\mathrm{n}, i}\right) \\
A^{\mathrm{ll}}
\end{array}\right), \quad A_{t}^{i}=\left(\begin{array}{cc}
A^{\mathrm{nn}} & A_{t}^{\mathrm{nl}, i}\left(x_{t}^{\mathrm{n}, i}\right) \\
0 & A^{\mathrm{ll}}
\end{array}\right), \\
& B_{t}^{i}=\left(\begin{array}{c}
B_{t}^{\mathrm{n}, i}\left(x_{t}^{\mathrm{n}, i}\right) \\
B_{t}^{\mathrm{l}, i}\left(x_{t}^{\mathrm{n}, i}\right)
\end{array}\right), \quad G_{t}^{i}=\left(\begin{array}{cc}
G_{t}^{\mathrm{n}, i}\left(x_{t}^{\mathrm{n}, i}\right) & 0 \\
0 & G_{t}^{\mathrm{l}, i}\left(x_{t}^{\mathrm{n}, i}\right)
\end{array}\right) .
\end{aligned}
$$

The nonlinear states can now sampled according to

$$
x_{t+1}^{\mathrm{n}, i} \sim \mathcal{N}\left(\bar{x}_{t+1}^{\mathrm{n}, i}, \bar{P}_{t+1}^{\mathrm{nn}, i}\right),
$$

and the linear states can be updated according to

$$
\begin{aligned}
& x_{t+1}^{\mathrm{l}, i}=\bar{x}_{t+1}^{\mathrm{l}, i}+\left(\bar{P}_{t+1}^{\mathrm{nl}, i}\right)^{\top}\left(\bar{P}_{t+1}^{\mathrm{nn}, i}\right)^{\dagger}\left(x_{t+1}^{\mathrm{n}, i}-\bar{x}_{t+1}^{\mathrm{n}, i}\right), \\
& P_{t+1}^{i}=\bar{P}_{t+1}^{\mathrm{ll}, i}-\left(\bar{P}_{t+1}^{\mathrm{nl}, i}\right)^{\top}\left(\bar{P}_{t+1}^{\mathrm{nn}, i}\right)^{\dagger} \bar{P}_{t+1}^{\mathrm{nl}, i} .
\end{aligned}
$$

6. Set $t:=t+1$ and iterate from step 2 .

In $[15,16]$ a maximum a posteriori estimate for the particle filter (PF-MAP) has been derived, which is argued to give a better point estimate in multi-modal applications. The PF-MAP estimate is an approximation of the MAP estimate given by

$$
\widehat{x}_{t \mid t}^{\mathrm{MAP}}=\underset{x_{t}^{i}}{\arg \max } p\left(y_{t} \mid x_{t}^{i}\right) \sum_{j} p\left(x_{t}^{i} \mid x_{1: t-1}^{j}\right) w_{t-1}^{j} .
$$

Following a similar reasoning, the RBPF-MAP estimate, can be shown to be

$$
\widehat{x}_{t \mid t}^{\mathrm{MAP}}=\underset{x_{t}^{\mathrm{n}, i}, x_{t}^{1, i}}{\arg \max } p\left(y_{t} \mid x_{t}^{\mathrm{n}, i}, x_{t}^{\mathrm{l}, i}\right) \sum_{j} w_{t-1}^{j} \mathcal{N}\left(x_{t}^{i} ; \bar{x}_{t \mid t-1}^{j}, \bar{P}_{t \mid t-1}^{j}\right),
$$

where $\bar{x}_{t \mid t-1}^{j}$ and $\bar{P}_{t \mid t-1}^{j}$ can be obtained from (13). Note that since our problem does not have a KF measurement update, instead of the commonly used double subscript denoting the time for the linear states, Algorithm 1 only uses a single subscript.

When implementing this in step 2 of the Algorithm 1, it needs 
to be taking into account that the covariance matrix $\bar{P}_{t}^{j}$ is rank deficient due to the presence of quaternion states. Because computation of (17) is computationally heavy, it could also be considered to use the most probable particle of the posterior. This would lead to similar results in Section 4.

\section{EXPERIMENTAL RESULTS}

\subsection{Experimental setup}

An experiment has been performed in which the magnetic field is generated by a magnetic coil where the number of windings $N_{w}$ is equal to 50, the current $I$ through the coil is $1 \mathrm{~A}$ and the radius $a$ of the coil is $6 \mathrm{~cm}$. A MEMS IMU (Xsens MTi) providing synchronized inertial and magnetometer measurements at a sampling frequency of $100 \mathrm{~Hz}$ is used. A picture of the experimental setup can be found in Figure 2. Ground truth data is collected from an optical reference system (Vicon system) and is used for validation of the estimates as well as for determining the position and orientation $R^{\mathrm{em}}$ of the coil.

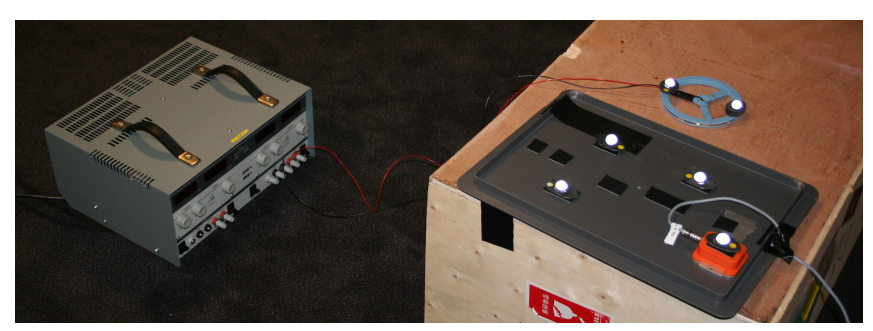

Fig. 2. The experimental setup consisting of an IMU (orange box), a coil and a power supply. Optical markers are present, used for obtaining ground truth data, via an optical reference system.

Before the magnetometer measurements can be used in Algorithm 1, they need to be preprocessed for two reasons. First, the model (9) assumes that the magnetometer only measures the magnetic field due to a coil. A constant term representing the local earth magnetic field therefore needs to be determined and subtracted from all measurements. Second, the IMU used outputs magnetometer measurements in arbitrary units, while the model (9) determines the magnetic field in Tesla. A constant multiplication on all axes is therefore needed. Both constants are obtained by determining a best estimate from a part of the data where the magnetic disturbance is (approximately) zero. The preprocessed data is illustrated in Figure 1. The circles represent the preprocessed magnetometer measurements, downsampled to $4 \mathrm{~Hz}$. The color of the circles represents the magnitude of the magnetic field. The magnetic field falls off cubically with distance which explains why the magnitude of the magnetic field is reduced quickly with distance from the coil. Each preprocessed measurement also gives rise to a red arrow indicating the direction of the magnetic field. The length of the arrows illustrates the magnitude.

\subsection{Results}

Using the collected inertial and magnetometer data, Algorithm 1 can be applied to obtain state estimates. Due to the fact that the magnitude of the magnetic field falls off cubically with distance, all results in this section are based on data no further away from the coil's origin than $40 \mathrm{~cm}$. These have been compared to the ground truth data from the reference system. This section focuses on analysis of the position estimates. Due to the unobservability discussed in Section 2.3 we do not expect exact matches between the RBPF estimates and the ground truth data. A good comparison of the quality of the estimates, however, are the radial position and height estimates. The error plots can be found in Figure 3. The RBPF is initialized around the true estimate using the reference data, but any other (reasonable) initialization will give comparable results.

As can be seen in Figure 3, very good position estimates are obtained. However, at approximately $42 \mathrm{~s}$, there is a big peak in both the radial position and the height errors. This can be explained by the fact that at this time instant, the sensor is the furthest away from the coil, almost $40 \mathrm{~cm}$. The approach presented in this work is thus able to obtain high accurate position estimates for longer times, only when the sensor remains close to the coil. This is a major limitation in using the magnetic field as a source of position information in the way presented in this paper. The further away from the magnetic disturbance the less informative the measurements become. Even though at $40 \mathrm{~cm}$ from the coil the signal to noise ratio is still good, tracking problems occur due to model errors. It is therefore important to have a good model of the magnetic field [17].
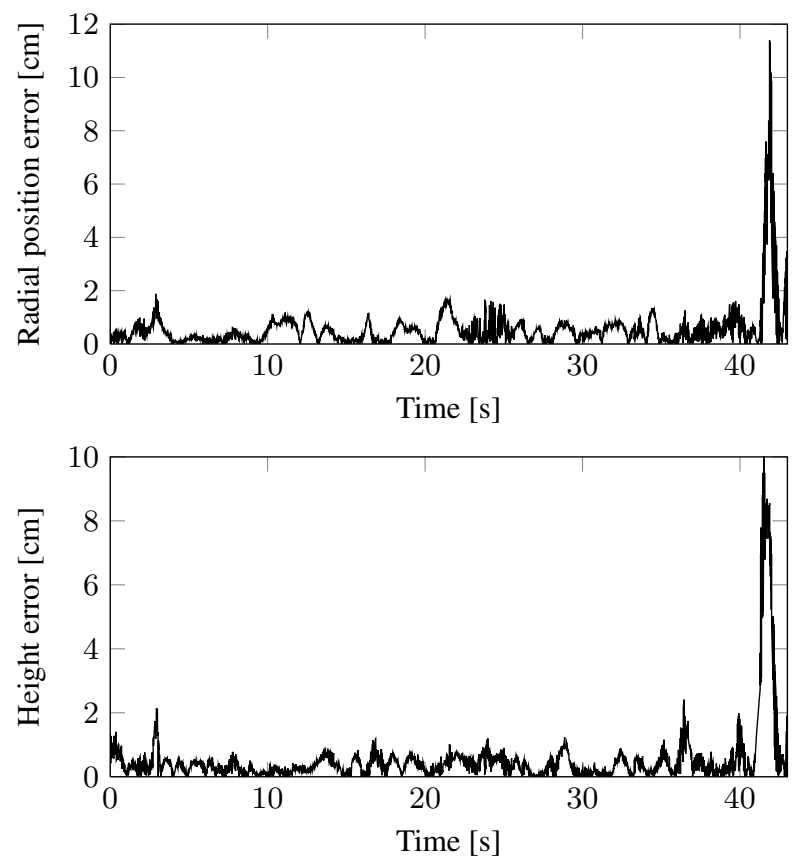

Fig. 3. Error plots comparing the RBPF position estimates with the ground truth data from the optical reference system.

\section{CONCLUSIONS AND FUTURE WORK}

This paper has shown that close to a magnetic distortion generated by a magnetic coil, good position and orientation estimates can be obtained from inertial and magnetometer data only. Ideas for future work include extending the magnetometer model to a more realistic measurement model. First trials show that we can probably deal with including the local earth magnetic field. We also aim at combining this work with [17] into an approach where simultaneous localization and mapping (SLAM) is possible. Another future line of research aims at studying the unobservability manifolds from the magnetic field in different cases.

\section{ACKNOWLEDGEMENTS}

The authors would like to thank Xsens Technologies for their support in starting this work as well as in collecting the data sets and Dr. Slawomir Grzonka for pointing out this interesting field of research. 


\section{REFERENCES}

[1] J.D. Hol, Sensor Fusion and Calibration of Inertial Sensors, Vision, Ultra-Wideband and GPS, Ph.D. thesis, Linköping University, Sweden, June 2011, Thesis No. 1368.

[2] S. Suksakulchai, S. Thongchai, D.M. Wilkes, and K. Kawamura, "Mobile robot localization using an electronic compass for corridor environment," in Proceedings of the IEEE International Conference on Systems, Man, and Cybernetics (SMC), Nashville, USA, October 2000, vol. 5, pp. 3354-3359.

[3] D. Navarro and G. Benet, "Magnetic map building for mobile robot localization purpose," in Proceedings of the IEEE Conference on Emerging Technologies and Factory Automation (ETFA), Mallorca, Spain, September 2009, pp. 1-4.

[4] I. Vallivaara, J. Haverinen, A. Kemppainen, and J. Roning, "Magnetic field-based SLAM method for solving the localization problem in mobile robot floor-cleaning task," in Proceedings of the 15th International Conference on Advanced Robotics (ICAR), Tallinn, Estonia, June 2011, IEEE, pp. 198203.

[5] E. Georgiou and J. Dai, "Self-localization of an autonomous maneuverable nonholonomic mobile robot using a hybrid double-compass configuration," in Proceedings of the 7th International Symposium on Mechatronics and its Applications (ISMA), Sharjah, United Arab Emirates, April 2010, IEEE, pp. $1-8$.

[6] D. Vissière, A.P. Martin, and N. Petit, "Using magnetic disturbances to improve IMU-based position estimation," in Proceedings of the European Control Conference (ECC), Kos, Greece, July 2007, pp. 2853-2858.

[7] E. Dorveaux, T. Boudot, M. Hillion, and N. Petit, "Combining inertial measurements and distributed magnetometry for motion estimation," in Proceedings of the American Control Conference (ACC), San Francisco, USA, June 2011, IEEE, pp. 4249-4256.

[8] J. Chung, M. Donahoe, C. Schmandt, I.J. Kim, P. Razavai, and M. Wiseman, "Indoor location sensing using geo-magnetism," in Proceedings of the 9th international conference on Mobile systems, applications, and services (MobiSys), Bethesda, USA, June 2011, ACM, pp. 141-154.

[9] "IndoorAtlas," http: / / www. indooratlas. com.

[10] B. Gozick, K.P. Subbu, R. Dantu, and T. Maeshiro, "Magnetic maps for indoor navigation," IEEE Transactions on Instrumentation and Measurement, vol. 60, no. 12, pp. 3883-3891, 2011.

[11] D. Törnqvist, Estimation and Detection with Applications to Navigation, Ph.D. thesis, Linköping University, November 2008, Thesis No. 1216.

[12] H.M. Schepers, Ambulatory assessment of human body kinematics and kinetics, Ph.D. thesis, University of Twente, Enschede, June 2009.

[13] D.J. Griffiths, Introduction to electrodynamics, vol. 3, Prentice Hall, New Jersey, 1999.

[14] F. Lindsten, Rao-Blackwellised particle methods for inference and identification, Licentiate thesis, Linköping University, Sweden, 2011, Thesis No. 1480.

[15] H. Driessen and Y. Boers, "MAP estimation in particle filter tracking," in IET Seminar on Target Tracking and Data Fusion: Algorithms and Applications, 2008, pp. 41-45.

[16] S. Saha, Y. Boers, H. Driessen, P.K. Mandal, and A. Bagchi, "Particle based MAP state estimation: A comparison," in 12th International Conference on Information Fusion. IEEE, July 2009, pp. 278-283.
[17] N. Wahlström, M. Kok, T.B. Schön, and F. Gustafsson, "Modeling magnetic fields using Gaussian processes," in Proceedings of the 38th International Conference on Acoustics, Speech, and Signal Processing (ICASSP), Vancouver, Canada, May 2013. 